\title{
La formación inicial en la enseñanza de la Historia. El patrimonio histórico-cultural en tercer grado de educación primaria
}

\author{
Initial training in the teaching of history. Historical-cultural \\ heritage in the third grade of primary education
}

DOI: https://doi.org/10.32870/dse.v0i23.787

\section{Francisco Hernández Ortiz*}

\begin{abstract}
Resumen
Este trabajo deriva de los resultados finales de una investigación cualitativa, con perspectiva fenomenológica, interpretativa-crítica. El método utilizado fue un estudio de caso. El sujeto de la investigación es un estudiante en formación inicial para la docencia, quien va construyendo sus propias nociones de enseñanza de patrimonio histórico-cultural. Por medio de una intervención pedagógica, logra que los niños de tercer grado de educación primaria se apropien de la noción del concepto de patrimonio histórico-cultural. Las técnicas e instrumentos utilizados fueron: la entrevista, el análisis hermenéutico de su documento recepcional y los datos de la propuesta de intervención. El objetivo fue identificar el proceso de formación inicial de un estudiante para profesor de educación primaria desde la intervención pedagógica con el tema de patrimonio histórico-cultural con niños de tercer grado de educación primaria. Los resultados muestran que sí es posible construir rasgos cualitativos de la formación docente desde una perspectiva sociocultural, enmarcada en un modelo integral de formación de docentes.
\end{abstract}

Palabras clave: formación inicial de profesores - enseñanza de la historia - patrimonio cultural - enfoque sociocultural.

\begin{abstract}
This work is derived from the final results of qualitative research conducted with a phenomenological and interpretative-critical approach and a case study method. The research subject is an individual in initial training for teaching, who builds his own notions of teaching about cultural historical heritage and, through pedagogical intervention, helps children in the third grade of primary education appropriate the concept of cultural-historical heritage. The technique and instruments used were the interview and hermeneutic analysis of his graduation thesis. The aim was to identify the initial training process of a primary education
\end{abstract}

* Doctor en Humanidades y Artes. SNI I, Asociado al Consejo Mexicano de Investigación Educativa. Cuerpo académico: Historia, educación y formación docente. Líneas de investigación: historia de la educación, historia cultural, procesos de formación docente, enseñanza de la historia, lenguaje y la comunicación. Profesor-Investigador, Benemérita y Centenaria Escuela Normal del Estado de San Luis Potosí. México. fhernandez@beceneslp.edu.mx 
teacher through pedagogical intervention on teaching third grade children of primary education about their cultural heritage. The results of the intervention show that it is possible to build qualitative features of teacher training from a sociocultural perspective in the framework of a comprehensive model of teacher training.

Key words: initial teacher training - history teaching - cultural heritage - sociocultural approach.

\section{Introducción}

El campo temático sobre el patrimonio histórico-cultural es interdisciplinario; ha sido objeto de investigación por la antropología, arqueología, sociología e historia. Giménez (2005) hace un análisis del concepto de patrimonio, denota que este tiene un significado polisémico. Concluye que este se asocia con la definición latina de patrimonium que, según el derecho romano significa: bien que se posee por herencia o legado familiar. En la modernidad es considerado como un bien común. El mismo Giménez, lo agrupa en la sociología cultural y lo define como bien común, creado por la sociedad. El patrimonio es un corpus representativo y simbólico de la cultura. Al mismo tiempo, es una forma de objetivación de la memoria y referente de la identidad. Se asocia a los bienes culturales de un pueblo o comunidad.

Los gobiernos, a través de la legislación y de políticas públicas, buscan preservar el patrimonio histórico-cultural. En cuanto tema educativo, es de interés de la pedagogía y la educación, desde estas dos disciplinas se busca conocer cómo los niños y jóvenes se apropian del concepto de patrimonio y de su preservación. A través del currículo es objeto de estudio en las asignaturas de Historia en la educación básica, y en educación superior es estudiado desde perspectivas multidisciplinarias. El trabajo que aquí se presenta se ubica en el campo de la pedagogía y educación; abona a la discusión de la necesidad de formar profesionales para la educación básica, con énfasis en el estudio de temas históricos y su enseñanza.

A continuación, se hace un análisis lacónico de algunas investigaciones sobre cómo los niños construyen las nociones conceptuales de patrimonio, donde el contexto social, cultural y su capacidad cognitiva son determinantes en la interpretación, comprensión, significado y representación del patrimonio histórico. Se concatenan algunos trabajos sobre el tema de patrimonio histórico-cultural, situados en la enseñanza de la historia desde la formación inicial de docentes para la educación básica.

Las nuevas perspectivas sobre el estudio del patrimonio histórico-cultural en la escuela de educación básica son relativamente recientes; los hallazgos han incidido en los procesos de enseñanza y aprendizaje. Así lo demuestran investigaciones como la de Vosniadou (2013), quien argumenta que los niños razonan los conceptos científicos basándose en modelos mentales, como parte de su proceso intuitivo para llegar a la construcción de los conceptos teóricos de

Diálo@os sobre Educación año 12 | número 23 | julio-diciembre 2021 | ISSN 2007-2171 
la ciencia. En el caso de las ciencias sociales, en específico en la historia, la construcción de conceptos como el de patrimonio histórico-cultural parte de los referentes o nociones que tiene cada niño, para luego confrontarlos con la construcción teórica formal del concepto que define al patrimonio; aquí, cada niño demuestra su saber, que luego reconstruye al adquirir el concepto formal. El contexto, para Saxe (2015), es el sitio donde se dan las prácticas cotidianas de interacción con la cultura, así es como las personas se van apropiando de los contenidos culturales; aunque depende también de la capacidad cognitiva de cada persona para interiorizar los significados y representaciones de los hechos históricos, científicos o culturales. Carretero y Montanero (2008) realizan estudios sobre la enseñanza de la historia considerando dos dimensiones: la dimensión cognitiva se refiere a las habilidades y recursos didácticos para aprender a pensar históricamente; la dimensión cultural explica la construcción de la identidad y trasmisión de la memoria colectiva. Al respecto, el patrimonio histórico-cultural es también una apropiación cultural que hacen los niños con la influencia del contexto familiar, social y comunicativo, en conjunto influyen para que los niños se apropien del conocimiento cultural.

El espacio formal de apropiación del conocimiento y la cultura es la escuela. Al respecto, Claxton (2014) lo denomina aprendizaje epistémico, porque debe estar centrado en actividades para pensar, aprender y conocer. Los niños desarrollan una mentalidad epistémica que les permite abordar la complejidad, la incertidumbre y la utilidad de los contenidos culturales. Desde la perspectiva sociocultural, la escuela propicia formas de trabajo donde los alumnos en colaboración aprenden y el docente solo es mediador, acercando el conocimiento a la zona de desarrollo próximo de sus alumnos.

Los estudios e investigaciones sobre las nociones o conceptos que construyen los niños sobre el patrimonio histórico-cultural es un campo en exploración. En Argentina, el estudio empírico de Lozano (2014) concluye en la necesidad de establecer una relación entre educación formal y patrimonio histórico-cultural, para interrelacionar el trabajo pedagógico y educar a los niños para su valoración, cuidado y preservación. En México, la investigación de Rodríguez (2019) documenta las nociones de patrimonio histórico-cultural que construyen los niños de educación primaria desde la experiencia social y cultural cercana a la localidad donde viven; demuestra que, como punto de referencia para iniciar el estudio formal del tema, se debe partir de lo que saben y conocen los estudiantes. Martín (2015) expone una experiencia empírica y pedagógica de los niños de educación infantil a través de un proyecto didáctico, demostró que adquieren interés, hábitos de cuidado y respeto hacia el patrimonio histórico-cultural.

El estudio de Solís (2019) sobre los niños y el patrimonio, con perspectiva antropológica y etnográfica, obtuvo resultados de cómo los niños se convierten en conocedores y defensores del patrimonio cercano a su experiencia de vida y a su contexto. Concluye que los niños logran desarrollar la creatividad e innovación al aprender entre los mismos niños y con los adultos; es una experiencia social y cultural en la apropiación del concepto de patrimonio histórico-cultur- 
al. La formación docente debe integrar estas nuevas formas de acceder al conocimiento cultural de forma creativa e innovadora, rescatando en todo momento la experiencia sociocultural que tiene cada niño o niña en las aulas.

El rol del profesor es fundamental en la construcción del conocimiento. En la perspectiva sociocultural, es mediador entre el conocimiento y las estructuras cognitivas de los estudiantes. El estudio de Veraksa (2016), desde la teoría vigotskiana y la práctica educativa, abunda en el valor de la comunicación entre maestro y niño en las etapas de la educación infantil. Relaciona teoría y práctica profesional en quienes se están formando para ser profesores. La formación en la práctica se traduce en acciones pedagógicas para que los alumnos de la educación básica construyan aprendizajes epistémicos, para aprender, saber y conocer. Quienes estudian para ser profesores de educación básica, su formación teórica es base para la intervención pedagógica; aproximan el conocimiento a los alumnos de preescolar, primaria o secundaria, a través de su enseñanza generan aprendizajes, consideran la zona de desarrollo próximo e integran andamiajes; esto provoca que se desarrollen procesos cognitivos que favorezcan la comprensión, reflexión y conocimiento en sus alumnos que implica una práctica docente inclusiva, participativa y reflexionada.

La formación inicial de profesores, en el contexto actual de la globalización, requiere, según Monereo (2013), avanzar hacia un modelo de formación integral; indagar en los procesos de construcción de nuevos aprendizajes, la identidad profesional y la participación del colectivo docente en el proceso formativo. Aunado a la construcción del conocimiento y el desarrollo de competencias generales y específicas para la docencia, actitudes motivacionales y recursos para la enseñanza, con apoyo de la investigación, la integración de los espacios para la reflexión y el análisis del trabajo docente, como las reuniones colegiadas, las prácticas educativas en las escuelas de formación y la posibilidad de investigar sobre su propia práctica docente.

El modelo de formación integral tiene matices de una configuración sociocultural del conocimiento y del aprendizaje. Está constituido por "la teoría Psicogenética de Piaget, el aprendizaje significativo de Ausubel y la perspectiva sociocultural de Vygotsky" (Reyes, 2007: 151). Aquí se conceptúa el conocimiento como un proceso continuo en el que influye lo individual, social y cultural, tanto para el aprendizaje como para la enseñanza. Los ambientes de aprendizaje situados, contextualizados son los referentes para que el profesor favorezca experiencias de aprendizaje significativo para los alumnos. La formación docente necesita modelos de intervención pedagógica con perspectiva sociocultural, considerando la formación teórico-disciplinar y una práctica pedagógica innovadora.

En síntesis, se avanza hacia un modelo de formación docente integral en donde se relacionan teoría y práctica pedagógica. De esta relación se genera un proceso dialéctico en la construcción de la teoría disciplinar y pedagógica, base teórica de las disciplinas científicas y las prácticas pedagógicas en la formación de los docentes. El Estudio de caso que aquí se

Diálo@os

sobre Educación

TEMAS ACTUALES EN INVESTIGACION : año 12 | número 23 | julio-diciembre 2021 | ISSN 2007-2171

4 
presenta, sobre el patrimonio histórico-cultural desde la experiencia de la formación de un licenciado en educación primaria, es congruente con esta perspectiva de modelo integral de la formación docente en México, dentro del marco de la globalización.

\section{Metodología}

La investigación es cualitativa y con una perspectiva fenomenológica, interpretativa-crítica; considera al sujeto de la investigación como un individuo que se encuentra en proceso de formación inicial, él mismo va construyendo sus propias nociones de enseñanza del patrimonio histórico-cultural y de los conceptos históricos. La naturaleza de la realidad donde realiza su trabajo docente es dinámico, holístico, divergente, múltiple e histórico: esto determina la complejidad que se manifiesta desde el sujeto de la investigación, el contexto, lo disciplinar y el grupo escolar donde se realiza su práctica pedagógica.

Los supuestos de la investigación: 1) La profundización en los conceptos teóricos de patrimonio histórico-cultural y conceptos históricos son fundamentales en el diseño de la planeación didáctica por el alumno que estudia para ser profesor. 2) El diseño de estrategias didácticas con una fundamentación socioconstructivista favorece que los niños se apropien de los conceptos de patrimonio histórico-cultural y de los conceptos históricos. 3) El estudiante para el profesorado, cuando reflexiona la enseñanza, fortalece su formación profesional.

El diseño de la investigación trazó que: la relación sujeto-objeto está íntimamente relacionada con los actores que intervienen directa e indirectamente en la investigación, donde se manifiestan valores subjetivos y las nociones individuales que tiene cada uno de los que intervienen en el proceso investigativo. Se reconoce la trascendencia que tiene la relación de la teoría y la práctica como un proceso dialéctico de retroalimentación para la mejora y transformación de la acción pedagógica.

El método de estudio de caso, permitió caracterizar al sujeto de la investigación: un estudiante en formación docente del séptimo y octavo semestres de la carrera de licenciado en educación primaria del ciclo escolar 2018-2019, de la Benemérita y Centenaria Escuela Normal del Estado de San Luis Potosí (México). Las técnicas utilizadas fueron: entrevista al estudiante para rescatar datos sobre el proceso de formación didáctica y disciplinar del tema de patrimonio histórico-cultural; se hizo un análisis hermenéutico de su informe de tesis, debido a que este es un documento que él construyó simultáneamente, como consecuencia de su formación desde la práctica profesional. Dicho documento sintetiza el proceso de diseño, aplicación y resultados de su propuesta de intervención pedagógica sobre el tema de patrimonio histórico-cultural.

El objetivo de esta investigación fue: identificar el proceso de formación inicial de un estudiante para profesor de educación primaria desde la intervención pedagógica, con el tema de patrimonio histórico-cultural con niños de tercer grado de primaria.

La investigación ayuda a profundizar en el proceso de formación docente, la práctica profesional, el análisis y reflexión de los procesos de formación desde la Escuela Normal. Los es- 
tudiantes que se forman para ser profesores en educación primaria aprenden a transformar la realidad en donde se sitúa su trabajo docente; nos referimos al aula, a los contenidos educativos sobre patrimonio histórico-cultural del estudiante en formación docente y el de sus alumnos de tercer grado, que atendió durante la jornada de práctica profesional que realizó en una escuela primaria de organización completa en la ciudad de San Luis Potosí.

El proceso de asesoría al estudiante en la elaboración de su tesis, el diálogo sobre los tópicos teóricos y didácticos de su práctica profesional, el análisis hermenéutico de su tesis y la entrevista, fueron fuentes de información y datos que demuestran que sí es posible mejorar la formación de docentes. El trabajo de investigación se ubica en el contexto actual de la formación docente; recupera información en un campo específico de la enseñanza de la historia en la escuela primaria, con el tema de patrimonio histórico-cultural; servirá de referencia para otros trabajos de investigación, considerando que aún falta un largo recorrido para la investigación en la enseñanza de la historia.

\section{Resultados}

En esta sección se presentan los resultados del análisis del diseño y de la propuesta didáctica que aplicó el estudiante en formación docente a los niños de tercer grado de primaria con el tema de patrimonio histórico-cultural. Consideró un primer componente: la fundamentación teórico-pedagógica como marco de referencia para el diseño de las secuencias didácticas, actividades de enseñanza y de aprendizaje. Este primer componente teórico-pedagógico lo apoyó en el constructivismo y socioconstructivismo. Los conceptos teóricos fueron la base de sus argumentaciones siguientes:

Interacción sujeto-objeto. El estudiante justifica atinadamente la relación sujeto-objeto desde la perspectiva constructivista; el sujeto en sus nociones o concepciones y que el objeto es aprehendido de forma distinta; por tanto, reconoce que es fundamental dicha relación en la construcción del conocimiento sobre los conceptos de patrimonio histórico-cultural en los estudiantes. El estudiante refiere:

La interacción directa con el patrimonio histórico-cultural se plantea como una oportunidad para que los estudiantes cambien sus concepciones (esquemas mentales) realicen acciones en pro de su conservación y se lleve a cabo la apreciación como parte del cambio histórico y la evolución cultural (TEN, 2019: 92). ${ }^{1}$

1 Tesis del Estudiante, realizada en el año 2019, sobre el tema de patrimonio histórico cultural con niños de tercer grado de educación primaria en una escuela de organización completa en la ciudad capital de San Luis Potosí. La versión final del documento se tituló "La apropiación del Patrimonio Histórico Cultural por los niños de tercer grado de educación primaria a través de estrategias didácticas". Tesis Licenciatura. Benemérita y Centenaria Escuela Normal del Estado de San Luis Potosí (BECENE), San Luis Potosí (México).

https://repositorio.beceneslp.edu.mx/jspui/handle/20.500.12584/307 
Esta noción conceptual del constructivismo del estudiante en formación para la docencia refleja una concepción activa de la construcción del conocimiento de niños y niñas de educación primaria.

La noción de aprendizaje sociocultural, la recupera de las aportaciones teóricas de Vygotsky y las transfiere a lo didáctico, reconociendo la experiencia social que tienen los niños sobre el patrimonio histórico-cultural; valora a la cultura como un dispositivo fundamental en la construcción de las nociones conceptuales de patrimonio. El aprendizaje sociocultural es un "referente para el diseño de la intervención docente al considerar las características del contexto que circunda la escuela primaria, específicamente los espacios y objetos histórico-culturales, experiencias de los alumnos fuera de la escuela" (TEN, 2019: 93). Esta reflexión del estudiante en formación, de la fundamentación de su propuesta, es pertinente con la afirmación de Vygotsky de que la educación es "una continuación del diálogo por el que se construye un mundo social de realidades" (Gómez, 1999: 3). Se apoyó en el aprendizaje sociocultural, en dicha propuesta demuestra que en la enseñanza debe discurrir lo social y lo cultural, como base para el diseño de las actividades y como punto de referencia para los aprendizajes de niños y niñas.

Incluyó el concepto de Zona de desarrollo Próximo (ZDP) de Vygotsky, como base de la mediación pedagógica entre el profesor y los alumnos. Argumenta que esto facilita a los "alumnos la apropiación del conocimiento histórico a través de la mediación del profesor, considerando el desarrollo cognitivo del alumno (andamiaje), su experiencia social, cultural y su interacción con el contexto socio-cultural" (TEN, 2019: 93). En síntesis, la "mediación, desarrollo cultural, afectividad, imitación e influencia del maestro, es propiamente un proceso en el que participan dos actores proactivos: el que aprende y el que coadyuva, valiéndose de medios o mediaciones diversas para que ocurra el aprendizaje" (Ruiz, 2015: 168).

El estudiante en formación docente es consciente que la enseñanza tiene una base teórica que requiere una planificación didáctica; reconoce los conocimientos previos que tienen los niños de tercer grado de la primaria sobre el patrimonio histórico-cultural, se apoya en las contribuciones de Ausubel sobre el aprendizaje significativo, reconoce que estos principios teóricos se convierten en el punto de inicio de toda actividad didáctica en el aula.

El Aprendizaje significativo, en la voz del estudiante es:

poner atención especial a los conocimientos previos del alumno para poder integrar los nuevos conocimientos, así formar nuevos esquemas mentales. Las nociones que tienen los alumnos sobre el patrimonio histórico cultural, la experiencia, consecuencia de la observación, ayudará a los estudiantes a reflexionar sobre el valor del patrimonio histórico cultural y sobre su bagaje cultural (TEN, 2019: 93).

La Autonomía del sujeto, es esencial en la construcción de conocimiento; al respecto, el estudiante para profesor asevera que "se espera que los alumnos desarrollen sus capacidades, interactuando, asimilando, incorporando nuevos conocimientos" (TEN, 2019: 94). Y son las estra- 
tegias didácticas el medio para apropiarse del conocimiento. El niño construye el conocimiento en la medida que interactúa con otros y comparte sus experiencias. La recuperación de los conocimientos previos que tienen los estudiantes, dando la oportunidad para que compartan lo que creen, saben o conocen sobre el patrimonio histórico-cultural, es condición para la planificación didáctica del estudiante que se forma para ser profesor de educación primaria. En la entrevista, este hace un ejercicio metarreflexivo:

las prácticas constructivistas y socioconstructivistas promueven un aprendizaje significativo, considerar los aprendizajes previos, aprender en diferentes contextos, trabajar colaborativamente, desarrollar la autonomía, interactuar con el objeto de estudio, cuestionarse, resolver problemas, entre otras acciones para desarrollar en los alumnos un sentido crítico, analítico y reflexivo (EJPR, 03/31/2020). ${ }^{2}$

Estos son los puntos esenciales considerados en la fundamentación de su propuesta didáctica con los niños de tercer grado de educación primaria.

\section{Los principios constructivistas en la enseñanza y aprendizaje de la historia} El sujeto de la investigación recupera algunos principios teóricos socioconstructivista y culturales en los que sustenta su propuesta para la enseñanza y aprendizaje de la historia, él expresa:

la construcción del conocimiento histórico está relacionada con las nociones de tiempo y espacio. De acuerdo con el plan de estudios con enfoque constructivista de la asignatura La entidad donde vivo se establece que en el enfoque didáctico que el desarrollo del pensamiento histórico se desarrolla a partir del conocimiento del tiempo histórico y el espacio geográfico (TEN, 2019: 94).

Los principios constructivistas de la planificación didáctica le dan un marco de referencia teórico a su intervención pedagógica. El estudiante para profesor, considera el valor que tienen el tiempo y espacio como categorías teóricas para sustentar a la historia y las enseñanzas de historia. Los conceptos de tiempo histórico y espacio, son básicos para situar la temporalidad en el devenir del ser humano, para entender cómo pasado y presente se correlacionan para comprender la evolución de la humanidad. Respecto al tiempo, es pertinente precisar que "la historia como ciencia que interpreta este pasado no ha aportado un modelo conceptual que nos permita elaborar un currículo para la enseñanza del tiempo histórico" (Pagés, 2010: 284).

El sujeto de la investigación sitúa el tema de patrimonio histórico-cultural dentro de la asignatura "La entidad donde vivo", esta corresponde al tercer grado de educación primaria. El propósito es que "los alumnos obtengan respuestas a los orígenes de su localidad" (SEP, 2012:

2 La entrevista fue realizada en marzo de 2020 al estudiante, quien en 2019 estaba terminando su carrera, y en 2020 es licenciado en educación primaria. 
2). Aquí se enuncian los conceptos históricos: tiempo histórico y espacio geográfico, esenciales para situar los hechos o circunstancias históricas en las que se erigieron los objetos culturales que son parte del patrimonio histórico-cultural cercano a la comunidad donde viven los alumnos. El estudiante en formación para profesor expresa que los niños de educación primaria deben intuir las nociones de tiempo y espacio para aprender el significado de patrimonio histórico-cultural.

\section{La historia e historiografía para situar el tema en la disciplina histórica}

En el apartado de la propuesta didáctica sobre los enfoques historiográficos y su incidencia en la enseñanza de la historia, aunque el estudiante lo aborda de forma fugaz, sí hace la aclaración de que su incorporación es para justificar que la enseñanza de la historia tiene una base científica que todo docente debe comprender.

Explica el enfoque positivista de la historia, no para transferirlo a la enseñanza sino para recuperar de este algunos elementos que son esenciales en la enseñanza, como el uso de las fuentes primarias; estas se relacionan con el concepto de Evidencia histórica, que significa tener certeza de que el objeto cultural fue creado o construido en determinado tiempo y espacio; es lo que pervive hasta la actualidad de ese hecho histórico.

El positivismo, plantea la idea de progreso, explica que el historiador, en este caso el docente, no debe interpretar, sino solamente acumular datos para describir objetivamente el suceso, por tanto, es necesario encontrar datos para construir la Historia. En este sentido será necesario abordar el estudio el tema histórico considerar el uso de diversas fuentes primaria en el aula o tener el mayor acercamiento posible (TEN, 2019: 95).

El enfoque historiográfico marxista, conceptúa a la historia como un hecho sociohistórico de lucha entre las clases sociales: Ios trabajadores y la burguesía. Es una historia crítica, cuestionadora de las estructuras hegemónicas que ostentan el poder económico y político. La superestructura económica da cohesión a las demás estructuras de orden social. Son evidentes dos clases: "una dominante que somete los instrumentos de producción y confisca al trabajador una parte de su trabajo y una clase dominada que solo tiene su fuerza de trabajo" (Corcuera, 1997: 65). El estudiante reconoce que comprender estas definiciones ayuda a que se entienda que no existe una historia única, y que la evidencia histórica que pervive es también consecuencia de la lucha de las clases sociales.

los hechos históricos podrán ser abordados enfatizando en que son consecuencia de la lucha social de los trabajadores en oposición a la burguesía. Se espera que dentro de las actividades planteadas se presente la crítica y el cuestionamiento hacia los hechos históricos, o especial, hacia las circunstancias, que han llevado a dar valor a ciertos espacios, objetos y monumentos culturales (TEN, 2019: 95). 
El tema de patrimonio histórico-cultural, desde la voz del estudiante, es interpretado como la construcción de los objetos culturales a través del tiempo, estos se han preservado hasta la actualidad, representan el poder económico o político de las elites gobernantes de la época.

El estudiante integró brevemente algunas contribuciones de cómo la Escuela de los Annales influyó en la disciplina de la historia; el rasgo multidisciplinar con "la geografía, economía y arqueología. La historia social recupera temas de obreros y trabajadores, movimientos y aquello de impacto social en el contexto escolar" (TEN, 2019: 96). Temas que habían sido invisibilizados por la historiografía positivista y marxista, aparecen en este nuevo enfoque historiográfico del siglo XX. El docente en formación logra asociar que en la enseñanza de la historia se interrelacionan los ámbitos social, económico, político y cultural para el estudio integral de los temas históricos.

Los ámbitos en la enseñanza de la historia ayudan a que los alumnos de educación primaria y los profesores sitúen un hecho histórico y lo relacionen con cada dimensión, para una mayor comprensión del tema histórico, objeto de estudio. El sujeto de la investigación llega a la siguiente conclusión: que el patrimonio histórico-cultural es producto de decisiones políticas, a las que subyace el factor económico y cultural de la época en la que fue creado el objeto cultural. Analizar el tema del patrimonio-cultural desde la asignatura de historia en la escuela primaria, ayuda a los alumnos y alumnas a valorar su pasado y a reconocer que el patrimonio es también consecuencia de las decisiones políticas, económicas, sociales y culturales de la época.

El estudiante en formación docente conceptúa que un hecho histórico no puede ser estudiado o analizado desde una sola perspectiva, sino que subyace también la subjetividad del historiador; esta es una característica del historicismo. Reconoce que este rasgo del historicismo es válido en la enseñanza de la historia ya que los alumnos pueden acceder a diferentes fuentes y formarse sus propios juicios de por qué fue o por qué sucedió. El tema de patrimonio-histórico cultural permite que "alumnos asuman en lo posible un papel de investigadores, que logren interpretar, usar las diversas fuentes y considerar elementos de la subjetividad; crear una mirada personal del Patrimonio Histórico-Cultural" (TEN, 2019: 96).

La enseñanza de la historia ayuda a que los alumnos de educación primaria accedan a fuentes, hagan observaciones de los objetos culturales cercanos a su localidad, y formen sus propios juicios, complementados con los del profesor y fundamentados en las fuentes históricas que consulte.

Con base en los puntos antes referidos, el estudiante elaboró su propuesta didáctica, diseñó y aplicó secuencias didácticas, identificó qué aprendizajes obtenendrían sus alumnos, las competencias a favorecer, además de la definición de las técnicas e instrumentos para llevar a cabo la evaluación del aprendizaje.

Es pertinente referir que, en los elementos de planificación, el estudiante integró contenidos del programa de la asignatura "La entidad donde vivo"; de acuerdo a los principios teóricos 
referidos con antelación, organizó el diseño de las secuencias didácticas, cuidó las fases inicio, desarrollo y cierre en el bosquejo didáctico. Los recursos y materiales didácticos también fueron considerados para cada una de las secuencias aplicadas. Expresa: "decidí fundamentar mi propuesta de intervención en tales corrientes pedagógicas debido a que son en las que se apoyan los actuales planes y programas de estudio" (EJPR,03/31/2020). Sin la consideración de algunas contribuciones de la historiografía, la enseñanza de la historia estaría incompleta ya que estudiar los hechos del pasado conlleva relacionar las categorías de tiempo, espacio, causalidad, cambio y permanencia para ubicar al hombre en el contexto sociohistórico, político y cultural del que forma parte.

\section{Indagación de las nociones de patrimonio histórico-cultural}

En esta sección se presentan los resultados de la aplicación de la secuencia didáctica, que tuvo como propósito recuperar los conocimientos previos de los alumnos de primaria sobre el patrimonio histórico-cultural. El estudiante en formación docente realizó una entrevista semi-estructurada a los niños de tercer grado de primaria con cuatro preguntas base, con la finalidad de rescatar las nociones sobre el concepto de patrimonio histórico para tener el referente de los conocimientos previos de los niños.

Elaboró tablas para clasificar los datos recabados por los instrumentos de los 37 alumnos de su grupo. El estudiante normalista tomó como base el concepto de patrimonio histórico-cultural: "conjunto de bienes, tanto materiales como inmateriales, acumulados a lo largo del tiempo. Estos bienes pueden ser de tipo artístico, histórico, paleontológico, arqueológico, documental, bibliográfico, científico o técnico." (Hechavarría, Piclín, 2010: 83); de la definición anterior, derivó el análisis de las respuestas de niños y niñas.

La primera pregunta que planteó ¿Sabes o has escuchado qué significa patrimonio histórico-cultural?, agrupó y generó cinco categorías de las respuestas: 1) sin nociones de patrimonio histórico-cultural; aquí ubicó a 15 niños cuyas respuestas se sintetizan en "No y nunca he escuchado"; 2) nociones alejadas del concepto, solo dos niños refirieron lo que a su juicio pensaban que significa, pero las respuestas no muestran o reflejan una noción acertada del concepto; "es cuando dos se enamoran y crean un hijo" o "lo más importante para SLP", ninguna de estas dos respuestas explican una noción del concepto antes expresado; 3 ) nociones de patrimonio asociadas a la familia, aquí se ubicaron siete niños cuyas respuestas se refieren a algún atributo de su familia como: "que proviene de los padres", "lo que se hereda de los padres", "la familia"; de estas respuestas el estudiante en formación docente demuestra que la construcción de las representaciones y significados de las cosas o los objetos, la primera fuente de información sin duda es lo que se aprende o se conoce en la familia; 4) nociones asociadas a la cultura e historia, cercanas al concepto teórico, aquí se ubicaron 10 alumnos, la noción se relaciona con la historia y la cultura; "antigüedades que heredan las personas, algo histórico o monumentos"; los niños 
lograron integrar en la definición algunos atributos que definen el patrimonio, aunque aun no es suficiente; 5) aproximación cercana al concepto identificando un lugar, espacio, monumento u objeto histórico, solo dos estudiantes lograron decir que el patrimonio histórico-cultural son los monumentos históricos más importantes, como la Caja del Agua (monumento histórico de la ciudad de San Luis Potosí).

La segunda pregunta realizada a los niños: ¿Conoces lugares y festividades que representan al patrimonio histórico-cultural de la ciudad de San Luis Potosí? La justificación de haber planteado esta pregunta tiene una base teórica pedagógica, al expresar que debían ser recuperados de la experiencia cercana a los estudiantes -cerca de la ubicación de la escuela primaria- a donde acudían los y las niñas de primaria a cargo del profesor en formación. Al mismo tiempo, porque la asignatura "La entidad donde vivo" contenía el tema para ser estudiado tomando como base lo cercano a los estudiantes, su comunidad, su ciudad y su estado. Es apropiado cuando el estudiante en formación argumenta y justifica las razones de haber planteado la pregunta 2, cuyos resultados son que, efectivamente, los y las niñas tienen conocimientos prácticos respecto al patrimonio histórico-cultural; aunque no logran definirlo conceptualmente, sí identifican algunos objetos culturales tangibles e intangibles.

Los 37 alumnos a los que se les aplicó el instrumento, de las seis opciones que tenían, eligieron varias, no solo una; esto es pertinente aclararlo: 26 ubicaron el monumento la Caja del Agua, esta fue durante el periodo colonial un lugar de almacenaje y distribución del agua que provenía de la sierra de San Miguelito, al sur de la ciudad. Actualmente está sin uso, solo es un monumento histórico representativo del periodo colonial. 12 alumnos señalaron el Centro de las Artes; este edificio originalmente fue un centro carcelario representativo del poder del porfiriato, y actualmente es museo y centro de formación artística. Otros 12 señalaron el Santuario de la Virgen de Guadalupe, una iglesia católica del periodo colonial, emblemática para los católicos, considerado un centro religioso. Una decena de estudiantes dicen que un lugar que pudiera ser considerado parte del patrimonio es "el lugar donde vivo"; esto es representativo considerar para un análisis profundo ya que puede estar asociado a una concepción holística o global del patrimonio como parte del entorno natural y social; aunque no lo logra expresar el estudiante en formación docente, tampoco los alumnos de la escuela primaria. Solo cinco niños dicen que el patrimonio cultural son las joyas de su familia, y cinco señalan la festividad de san Judas Tadeo; esta última asociación es valiosa, porque se aproxima a la representación y significado del patrimonio histórico-cultural intangible, con un carácter totalmente religioso, pero cercano al entorno donde viven los alumnos. En la pregunta 3. ¿Por qué crees que un lugar, espacio u objeto puede ser considerado patrimonio histórico-cultural? se pretendió conocer las nociones que tienen niños y niñas del significado de espacio, lugar u objeto para ser considerado patrimonio histórico-cultural. Esta tercera pregunta demuestra las nociones donde está de por medio el tiempo, el espacio y el valor histórico del objeto cultural; 12 no respondieron, cuatro expresan que nun- 
ca lo habían escuchado o simplemente no sabían. Respecto al espacio, solo cuatro tratan de relacionarlo con el concepto de patrimonio histórico; pero no se logra precisar en las respuestas "porque puede ser un espacio libre y natural", pues no dan mayor información. Siete expresan que el patrimonio histórico-cultural que ejemplifican está asociado con el tiempo (la Caja del Agua), y tres comentan que es todo "aquello que hace mucho tiempo sucedió". Dos dicen que es todo aquello importante para San Luis Potosí.

Para la pregunta 4. Escribe el nombre de un monumento o espacio u objeto cultural que conozcas y que consideres que es parte del patrimonio histórico-cultural, las respuestas fueron diferentes: 12 alumnos no respondieron, cuatro dicen que no saben o no lo recuerdan. 12 dicen que es la Caja del Agua, el resto, su respuesta no tiene relación con lo solicitado en la pregunta.

El estudiante en formación docente, a través de la planificación didáctica y utilizando técnicas de recolección de datos, logra documentar las nociones que tienen los niños de tercer grado sobre el patrimonio histórico-cultural. Los resultados de esta primera actividad demuestran que los niños tienen una noción que se aproxima a la definición del concepto, aunque cada niño la ha construido conforme a su experiencia y contexto sociocultural donde vive.

Los resultados de esta actividad de indagación de las nociones que tienen los alumnos sobre el tema facilitaron al estudiante en formación docente aplicar las secuencias didácticas para el estudio formal del tema por los niños de tercer grado de educación primaria.

\section{Los efectos de la secuencia didáctica en la generación de los aprendizajes sobre el patrimonio histórico-cultural}

La enseñanza planificada con datos objetivos permite al estudiante que se forma para ser profesor desarrollar una práctica docente sustentada y argumentada, con datos recabados a través de técnicas e instrumentos de la investigación aplicados a la práctica docente.

El trabajo colaborativo aparece como una condicionante para el aprendizaje de los alumnos. Colaboran, comparten e interactúan y llegan a la definición de las nociones de lo que significa, en los diferentes momentos donde el estudiante va aplicando cada una de las secuencias didácticas que componen su propuesta.

Las secuencias pretendían "que los alumnos compartieran información que previamente habían investigado en diferentes fuentes respecto al patrimonio y patrimonio histórico-cultural, para después construir colaborativamente un concepto y alguna representación de lo que definiera por patrimonio histórico-cultural" (TEN, 2019: 103). El estudiante en formación explica que la estrategia didáctica tiene un propósito, y marca la ruta a seguir en el proceso de enseñanza. La indagación por parte de niños y niñas de tercer grado de primaria es una primera acción que realizan para despertar su interés por el estudio del tema de forma colaborativa.

Las categorías analíticas definidas fueron: creación de ambientes de aprendizaje con trabajo colaborativo, interacciones entre los alumnos y el profesor en el proceso didáctico. 
1) Creación de ambientes de aprendizaje. Estos favorecen en los niños la apropiación del concepto de patrimonio histórico-cultural, deben ser acondicionados por el profesor para el trabajo en el aula. "El primer acercamiento con los alumnos se dio a través de la organización áulica, los pupitres se colocaron alrededor del aula para facilitar la interacción grupal y el espacio para trabajar en equipo" (TEN, 2019: 104). El estudiante justifica que sustentó en principios teóricos la creación de ambientes de aprendizaje; implicó crear en el aula condiciones materiales, didácticas, comunicativas y pedagógicas que facilitaran el trabajo individual y colaborativo. Los ambientes de aprendizaje se construyen,"su caracterización es variada y depende de la disposición intelectual, emocional y afectiva de cada sujeto, del mismo modo involucra las condiciones contextuales y materiales que afectan directamente al sitio donde los participantes entran en interacción" (Noyola, 2018: 98). Desde los preceptos de Vigostky, aprender juntos y compartir conocimiento se favorece desde los ambientes de aprendizaje, como sucedió en la indagación sobre el tema de patrimonio histórico-cultural que hicieron los alumnos de tercer grado de primaria.

2) El trabajo colaborativo. Fue la estrategia para que los alumnos cooperaran en el estudio del tema: "se definieron equipo de trabajo colaborativo para compartir de manera detallada la información que investigaron en una de las acciones que facilitó sobremanera el trabajo realizado fue que los alumnos dentro de sus equipos de trabajo eligieron un líder para que cuidara el trabajo en equipo" (TEN, 2019:105). El trabajo colaborativo dio la oportunidad a los niños para elegir un responsable de moderar y encauzar las actividades; fundamenta que en el "diseño de situaciones de colaboración entre pares, el docente debe considerar la interacción de diversos factores, tales como el grado de dificultad de la tarea, la comunicación al alumno de que su desempeño será evaluado y el género" (Castellaro, 2011: 140).

3) Interacciones para el aprendizaje. Son formas de colaborar con lo que cada niño sabe y le permite cooperar con el otro lo que investigó, lo que piensa o lo que representa sobre el patrimonio histórico-cultural, es decir "los alumnos comenzaron a compartir la información que investigaron y a poner en común las ideas que encontraban en sus investigaciones" (TEN, 2019: 104).

El constructivismo y el socioconstructivismo consideran que el aprendizaje se construye en colaboración, es de carácter social ya que cada persona, desde su experiencia social, construye conocimiento poniendo en práctica sus habilidades y actitudes; al respecto el estudiante normalista expresa: "el clima y orden de trabajo fue favorable, ya que los alumnos estaban enfocados en el trabajo, se distribuyó en cada equipo algún alumno con habilidades sobresalientes para el dibujo, para la reflexión, crítica y expresión oral" (TEN, 2019: 104).

Las interacciones constituyen las formas básicas de carácter social para intercambiar experiencia, conocimiento y aprender de los otros; esto coincide con la aseveración de que el aprendizaje tiene una "naturaleza social específica y un proceso mediante el cual los niños acceden a 
la vida intelectual de aquellos que les rodean" (Vygotsky,1979: 136). Lo anterior coincide con la reflexión que hace el estudiante normalista cuando expresa que los niños:

compartieran los puntos de vista personales, más allá de la investigación que habían realizado. Un alumno comentó, mientras se estaba abordando qué significaba el patrimonio familiar, que su abuelo tenía una casa muy grande y que cuando falleció la dejó en herencia para sus familiares, así como joyas y otros artículos que le pertenecían (TEN, 2019: 105).

Las categorías analíticas antes referidas recuperan la evidencia empírica de la forma en que los niños del tercer grado de educación primaria se van apropiando del concepto de patrimonio histórico-cultural, guiados por las secuencias didácticas aplicadas por el estudiante en formación docente.

\section{Una aproximación a la construcción de las nociones de patrimonio histórico-cultural por los alumnos de tercer grado de la escuela primaria} De la aplicación de la secuencia didáctica introductoria del tema de patrimonio histórico- cultural utilizada por el estudiante en formación docente, de acuerdo con las conclusiones que presentó de cada equipo colaborativo conformado por siete alumnos, se procedió a realizar un análisis, identificando los puntos que a continuación se enuncian como categorías analíticas de esta sección.

1) Definición del concepto de patrimonio histórico. Los integrantes de cada equipo lograron consensar una definición, resultado de lo indagado en fuentes bibliográficas; convienen en que es un conjunto de bienes materiales e inmateriales acumulados a través del tiempo, que pueden clasificarse en histórico, natural, material, inmaterial e intangible. Los niños lograron identificar que la naturaleza y la comida son parte del patrimonio cultural.

Un concepto más amplio de patrimonio histórico-cultural es la inclusión de las obras de arte, música, literatura, lengua, ritos, arquitectura; un equipo define que el patrimonio es una herencia de la humanidad, por tanto, una preocupación de la Organización de las Naciones Unidas para la Educación, Ciencias y Cultura (UNESCO). De acuerdo con las nociones de patrimonio histórico-cultural que expresan los niños, es reflejo de su construcción conceptual, así lo reconoce el estudiante en formación:

considero que debido a que los conceptos se fueron trabajando a lo largo de la sesión, los alumnos se sentían más identificados con el tema y ya no era el principal interés resolver dudas; además que las exposiciones fueron claras y concretas basándose en el material que elaboraron (TEN, 2019: 107).

2) La representación gráfica de la noción de patrimonio histórico-cultural. El estudiante normalista guio a los alumnos para que representaran gráficamente, por medio del dibujo, ejem- 
plos de patrimonio histórico-cultural, encontrando lo siguiente: al hacer una agrupación, hay quienes identificaron a la naturaleza poniendo un árbol. Otros dibujaron monumentos como la Caja del Agua, la Calzada de Guadalupe y el Museo Laberinto de las Ciencias y las Artes. Inmaterial, como las enchiladas y el chocolate. Es pertinente mencionar que todos los dibujos de las y los niños corresponden al patrimonio histórico-cultural de la ciudad, y los monumentos y edificios están cerca del entorno escolar. En el caso de la comida, el plato tradicional referido fueron las enchiladas potosinas y el chocolate La frontera, productos tradicionales de San Luis Potosí.

Al respecto, el estudiante en formación docente expresa que:

Los datos obtenidos de los productos de los alumnos permitieron definir lo que es el patrimonio histórico-cultural se haya dado desde diferentes perspectivas e ideas personales de los estudiantes, ya que comenzaron a vincular un concepto teórico (de acuerdo a su investigación) ligando con las nociones o construcciones previas, así mismo, relacionado con el contexto histórico y cultural que circunda su escuela, al realizar dibujos de monumentos y espacios históricos como la Caja del Agua, la Calzada de Guadalupe y comida perteneciente a la entidad (enchiladas potosinas), etc. Por lo tanto, las estrategias didácticas aplicadas en esta sesión ayudaron a que los estudiantes construyeran nuevo conocimiento (TEN, 2019: 107).

El estudiante normalista argumenta teórica y empíricamente con datos, justifica su discurso pedagógico sobre las nociones que las y los niños van construyendo del concepto en cuestión. El aprendizaje del "patrimonio histórico-cultural es un proceso que conlleva estudio, gusto y responsabilidad por el tema" (EJPR,03/31/2020). Respecto a la metarreflexión que hace sobre la aplicación de las secuencias didácticas refiere que estas:

se diseñaron tomando como punto de referencia el contexto que circunda su escuela primaria; esto favoreció el aprendizaje porque no se trataba de edificios, monumentos o espacios históricos ajenos a ellos, sino que el patrimonio histórico-cultural en el que se enfatizó fue el que al menos identificaban y después le encontraron sentido y desarrollarlo mayor apropiación (EJPR,03/31/2020).

El estudiante está convencido que las nociones que tenían los y las niñas del concepto se modificaron con la intervención didáctica que hizo a través de las secuencias didácticas y de acuerdo a los datos obtenidos:

Considero que las nociones de los alumnos fueron cambiando de una manera fluida y significativa; ya que se partió desde el hecho de entender qué es el patrimonio (desde los diferentes tipos de patrimonio) y después focalizar el patrimonio histórico cultural; identificarlo en el contexto que circunda su escuela primaria, interactuar de manera directa con él y ahí comprender con mayor ímpetu por qué es importante; finalmente expresar la experiencia con alguna creación artística propia y socializarla entre grupo y con la comunidad escolar (EJPR,03/31/2020).

Diólo@os 
La síntesis que hace el estudiante, basado en la teoría y los datos empíricos, exhibe la apropiación y construcción de su saber teórico y práctico sobre la enseñanza, el aprendizaje y del tema.

\section{El aprender a enseñar el tema de patrimonio histórico-cultural en la escuela primaria}

La formación inicial de profesores para la educación primaria, de acuerdo con la estructura curricular del plan de estudios, se agrupa en campos formativos: la experiencia pedagógica de la formación se ubica en el campo formativo de la práctica profesional y el de la formación disciplinar. A fin de tener datos del sujeto de la investigación, se elaboró una entrevista semiestructurada para conocer cuáles fueron los aprendizajes obtenidos por el estudiante; desde el inicio de la construcción de su propuesta didáctica, en su aplicación y su relación con el proceso de formación inicial en la Escuela Normal, a partir del campo de lo disciplinar en el tema del patrimonio histórico-cultural.

El sujeto de la investigación expresa que en la práctica profesional y en la elaboración de la tesis obtuvo aprendizajes sobre cómo enseñar, qué es aprendizaje, porqué se debe fundamentar teóricamente la enseñanza, de forma especial sobre el tema de patrimonio histórico-cultural.

aprendí que aquellos bienes tangibles e intangibles representan en gran medida lo que ahora somos como sociedad; si analizamos rigurosamente el patrimonio histórico y cultural de nuestra entidad podríamos entender las luchas sociales, políticas y económicas que se dieron en determinado tiempo histórico y por lo tanto en la actualidad, focalizar en los conceptos de empatía, causalidad, cambio, permanencia y otros más para mejorar como sociedad (EJPR,03/31/2020).

En el discurso del estudiante normalista derivado de la entrevista, refiere que el patrimonio histórico-cultural es también la herencia de los pueblos, este es testimonio de los que llegaron a ser y es reflejo de hacia dónde quiere llegar un pueblo o una sociedad. "Personalmente, en la construcción de mi trabajo de investigación aprendí un poco más sobre mi entidad y las riquezas históricas y culturales a las que podemos acceder con facilidad" (EJPR,03/31/2020). El conocimiento didáctico adquirido en la enseñanza del tema lo sintetiza de la siguiente manera:

aprendí que las estrategias didácticas que diseñé deben estar enfocadas especialmente en el aprendi-

zaje de los alumnos; si se pone en el centro a los alumnos, sus gustos, intereses, su contexto, los recursos y materiales didácticos y los demás agentes de la comunidad escolar que intervienen en el aprendizaje, se tiene como consecuencia un aprendizaje más significativo (EJPR,03/31/2020).

El estudiante en formación docente argumenta que para que se dé el aprendizaje, se requiere que los ambientes de aprendizaje sean los correspondientes, eso incluye los recursos 
materiales, mobiliario y espacio, implica también considerar la teoría y los antecedentes escolares del estudiante:

Tener en cuenta la teoría y el trabajo que otros profesores e investigadores han realizado con antelación es uno de los mayores aprendizajes en la construcción de mi trabajo; así, conociendo los hallazgos de otras investigaciones y las teorías que fundamentan el objeto de estudio (EJPR,03/31/2020).

Los estudios para licenciado en educación primaria se distribuyen en ocho semestres. La estructura curricular se compone de cinco campos de formación: psicopedagógico, preparación para la enseñanza y el aprendizaje, lengua adicional y tecnologías de la información y la comunicación, práctica profesional y cursos optativos.

Esta investigación cualitativa de un estudio de caso, se concentra en un estudiante que se forma profesionalmente a nivel licenciatura como profesor de educación primaria, la experiencia de formación docente se focaliza en los campos de preparación para la enseñanza y el aprendizaje, y en el de práctica profesional. Respecto al campo de práctica profesional, comprende ocho cursos semestrales; aquí el estudiante adquiere conocimientos básicos para comprender el contexto, el grupo escolar, los alumnos, los programas, la enseñanza y los procesos de aprendizaje.

El campo de preparación para la enseñanza y aprendizaje comprende 18 cursos, de los cuales hay tres que se agrupan en el estudio de la historia y su enseñanza; a fin de valorar cómo estos cursos proporcionaron bases para el estudio del patrimonio histórico-cultural, el estudiante normalista expresó que las asignaturas de Educación histórica en el aula y Educación histórica en diversos contextos, le ayudaron a comprender el valor formativo de la enseñanza de la historia en la educación primaria "en uno de los cursos mencionados realicé una investigación sobre el patrimonio histórico-cultural de la entidad de San Luis Potosí; considero que tal trabajo fue un antecedente importante para la realización de mi tesis sobre el patrimonio histórico-cultural" (EJPR,03/31/2020).

La acción pedagógica realizada por el estudiante normalista en la escuela primaria sintetiza la trascendencia de los alumnos en el proceso enseñanza-aprendizaje, el diseño didáctico, las condiciones en el aula y la influencia del contexto en la construcción de las nociones de patrimonio histórico-cultural.

\section{Discusión}

La propuesta didáctica elaborada por el estudiante normalista refleja la apropiación del conocimiento teórico adquirido a lo largo de los ocho semestres que componen la carrera de licenciado en educación primaria; explica con suficiencia los fundamentos de cómo se construye el conocimiento por las y los niños, apoyado en el constructivismo y socioconstructivismo. Su propuesta didáctica recu- 
pera conceptos teóricos fundamentales como: conocimiento, aprendizaje, aprendizaje significativo, zona de desarrollo próximo, andamiaje, contexto social y cultural. Es pertinente acotar que rescata los conceptos teóricos, y luego busca transferirlos para explicar lo pedagógico; esto es valioso ya que logra entender que la teoría es una construcción general explicativa de cómo se construye el conocimiento, pero no es específica para un tipo de conocimiento. Sin embargo, el normalista la adapta y la acuña para intentar explicar cómo se construyen las nociones de patrimonio histórico-cultural en los niños de tercer grado de educación primaria. Demuestra su importancia en la enseñanza, los conocimientos previos de los alumnos y de la experiencia social, esta, en congruencia con lo que interactúa o conoce el alumno y según el contexto donde vive. Lo anterior es coincidente con la perspectiva socioconstructivista de que toda enseñanza debe ser situada para que los aprendizajes sean significativos, o para que el andamiaje efectivamente cumpla su función de incidir en la zona de desarrollo próximo de cada uno de los alumnos.

Los referentes teóricos que plantea en su propuesta didáctica son los que le permiten al estudiante hacer una argumentación de su propia práctica educativa, y lo hace justificando porqué las secuencias didácticas tuvieron la organización de inicio, desarrollo y cierre, y cómo cada una de las actividades cumplieron su función de favorecer los aprendizajes. Es pertinente señalar que los datos que presenta el estudiante de los resultados de la aplicación de su propuesta permiten inferir la comprensión y utilización que hace de la teoría para explicar su intervención pedagógica, lo cual es propio para el contexto y entorno escolar donde realizó su práctica pedagógica. Esto es coincidente cuando se asevera que "la práctica docente en sí misma es una acción compleja, matizada por factores, propios del docente, el aula, el contexto y la cultura escolar" (Hernández, 2014: 1).

Tanto el análisis del diseño y los resultados de su aplicación demuestran que, en el proceso de formación de profesores, la teoría es fundamental para toda intervención didáctica. La práctica es la concreción de los referentes teóricos donde se manifiestan en la acción concepciones de aprendizaje, enseñanza y, desde luego, los conceptos científicos de la disciplina. La formación desde la práctica docente permite que el estudiante "aplique los diferentes conocimientos, habilidades, actitudes y valores, adquiridos, así como dar cuenta de su progreso" (Chapa, 2016: 161). El tema de patrimonio histórico-cultural corresponde al campo histórico situado en la historia social y cultural, tema que forma parte de los contenidos educativos de los planes y programas de estudio vigentes en la educación básica.

La innovación que hace el estudiante en formación docente al diseñar una propuesta, aplicarla, y luego recuperar datos sobre los efectos en el aprendizaje del tema por los y las niñas, le permitió deducir aprendizajes esenciales: el valor de la teoría, el diseño secuencial de las actividades, la experiencia social de cada uno de los niños, la influencia del entorno en el conocimiento del patrimonio histórico-cultural.

Los datos recabados del estudiante a través de la entrevista admiten aseverar que la formación docente está estructurada en el conocimiento de los sujetos del aprendizaje, que son 
los alumnos; eso implica el conocimiento de su desarrollo y cómo construyen el conocimiento; la teoría disciplinar que se refiere a campos del conocimiento muy específico de las ciencias sociales, naturales, matemáticas, humanidades y artes. Reconoce que en el campo de la enseñanza de la historia sí hubo asignaturas en el plan de estudios que le ayudaron a tener un acercamiento con el tema de patrimonio histórico-cultural, siendo motor de motivación para indagar y profundizar en el tema de investigación.

Acerca del campo de la formación desde la práctica pedagógica, el estudiante señala la necesidad de que las decisiones en el proceso de enseñanza y aprendizaje deben ser reflexionadas y sustentadas en la teoría. Esto coincide con la perspectiva de que el "proceso reflexivo deberá asumirse como una tarea sustantiva en la formación inicial del profesional de la educación, ésta tiene que estar presente a lo largo de la preparación académica" (Reyna et al., 2018: 42). La apropiación del conocimiento teórico se refleja en su discurso pedagógico al justificar sus formas y procedimientos en la intervención pedagógica en el estudio del tema de patrimonio histórico-cultural.

El tema estudiado, integrado a los planes y programas de estudio para la educación básica, tiene un carácter formativo en los niños; no con el propósito de que ellos se apropien de los conceptos teóricos que lo definen como resultado de la construcción cultural y evolución de una sociedad, sino para que en primera instancia reconozca y valore lo cercano a él, en su contexto. Los niños de tercer grado de primaria estudian la historia de su localidad, eso implica su colonia, ciudad, municipio y estado, por tanto, la experiencia vivida por el estudiante normalista demuestra que, efectivamente, sí es posible acercar a los estudiantes de primaria el tema de patrimonio histórico-cultural, e identificar que algunos niños sí tienen nociones; unos lo relacionan a los bienes de la familia como primer referente, otros lo asocian con lo cercano a su experiencia, como edificios, monumentos tradicionales de su localidad. Las actividades de las secuencias didácticas aplicadas también demuestran que, una vez que es dosificado el tema y planteado creativamente a los niños, estos alcanzan a apropiarse de las nociones del concepto de patrimonio histórico-cultural, pero también logran identificarlo en su contexto; por ende, esto puede favorecer el desarrollo de una conciencia histórico-social para preservar el patrimonio histórico-cultural de su localidad.

Si el patrimonio histórico-cultural es todo aquel objeto creado por la humanidad, ya sea para representar ideas, pensamientos o expresiones artísticas, materiales o inmateriales, es asimismo todo aquello relacionado con el hábitat del hombre; por tanto, puede ser también de carácter natural, simbólico y representativo de valor para la humanidad, por lo que su preservación se convierte en una necesidad para conservar la memoria histórica de un pueblo o sociedad. Los ejemplos de patrimonio histórico-cultural que rescatan los niños como resultado del trabajo de las secuencias didácticas diseñadas por el estudiante normalista, son representativos para los habitantes de la ciudad de San Luis Potosí; ante las argumentaciones que dan los niños 
se demuestra que los conocen, que los definen como algo del pasado que hay que preservar. Aunque este asunto de la preservación no logró abundarse con suficiencia en el proceso de trabajo didáctico, este puede ser motivo para una mayor profundización en estudios posteriores.

Una deducción que se hace es que los niños son capaces de construir las nociones de patrimonio histórico-cultural en la medida que el profesor y la escuela consideran estas temáticas como actividades lúdicas, pero, sobre todo, cercanas a los intereses de los alumnos, recuperando su experiencia social. El patrimonio histórico-cultural es también evidencia histórica, testimonio de la creación humana; es reflejo de lo que cambia y a la vez permanece a través del tiempo; por eso es un legado que hay que cuidar, proteger y preservar.

Respecto a la enseñanza de la historia en la educación primaria, se demuestra la importancia de los conceptos históricos como el tiempo y el espacio, esenciales para situar los objetos culturales que son parte del patrimonio histórico-cultural. El cambio y la permanencia, evidencia histórica y empatía, pueden ser considerados para generar una conciencia histórica en los estudiantes de educación primaria.

\section{Reflexiones finales}

El campo de estudio del patrimonio histórico-cultural, como objeto de enseñanza y aprendizaje para los alumnos de educación básica, es relativamente reciente; así lo constatan algunas investigaciones recuperadas en el estado del arte, sobre todo las de corte cualitativo con perspectiva sociocultural.

La formación docente, como proceso, está sostenido en una base teórica disciplinar que ayuda a la comprensión de los procesos de enseñanza-aprendizaje y al conocimiento del sujeto. La integración de los contenidos disciplinarios del sujeto del aprendizaje, el contexto y rol del profesor, son identificados por el estudiante en formación como ejes para que se complementen con la práctica pedagógica.

La práctica docente es una acción intencionada, reflexionada, fundamentada en la teoría; es una forma de confrontar la relación sujeto-objeto de conocimiento, donde ambos son modificados y transformados. La aprehensión de las características esenciales de los objetos o de las cosas, ayudan a la construcción de las nociones científicas; en este sentido, el estudiante en formación logró que sus alumnos construyeran las nociones de patrimonio histórico-cultural. Basado en una perspectiva teórica sociocultural, le da un valor al contexto, a los aprendizajes previos, a la mediación, a la zona de desarrollo próximo; logra utilizar y argumentar los conceptos históricos para estudiar el tema a mayor profundidad junto con los alumnos.

El estudiante es consciente de que el diseño didáctico de una propuesta de intervención fundamentada y argumentada facilita la apropiación del conocimiento por parte de los alumnos. El trabajo colaborativo rompe con el trabajo individual tradicional, para construir en los alumnos las nociones de aprendizaje social: juntos aprendemos en colaboración. Quedaron 
pendientes las estrategias para la preservación del patrimonio histórico, que puede ser motivo para que se continúe con otros trabajos de investigación.

\section{Referencias}

Carretero, M.; M. Montanero (2008). Enseñanza y aprendizaje de la historia: aspectos cognitivos y culturales. Cultura y Educación, 20(2), 133-142. https://www.tandfonline.com/doi/abs/10.1174/113564008784490361

Castellaro, M.; M. Dominino (2011). El proceso colaborativo en niños de escolaridad inicial y primaria. Una revisión de trabajos empíricos. Revista Intercontinental de Psicología y Educación, 13(2),119-145. https://www.redalyc.org/articulo.oa?id=802/80220774007

Chapa, M. (2016). El desarrollo de las competencias de investigación durante la formación inicial docente: de la observación y análisis de la práctica educativa a la tesis de investigación. En Guzmán Elizondo H. A. et al. (eds.). La formación inicial y continua de docentes. México: Fondo Editorial Nuevo León.

Claxton, G. (2014). School as an Epistemic Apprenticeship: The Case of Building Learning power/ La escuela como aprendizaje epistémico: el caso de construyendo el poder para el aprendizaje. Infancia y Aprendizaje, 37(2), 227-247.

Corcuera de Mancera, S. (1997). Voces y silencios en la historia siglo XIX y XX. México: Fondo de Cultura Económica.

Giménez, G. (2005). Patrimonio e identidad frente a la globalización. Patrimonio Cultural y turismo. Cuadernos, (13), 177-182.

Gómez, F.; R. Mejía Arauz (1999). Vygotsky: La perspectiva vygotskyana. Correo pedagógico, (4), 3-6. Hechavarría, R.; C. Piclín (2010). El desarrollo de la identidad cultural mediante el estudio del patrimonio histórico y su vínculo con la historia local. EduSol, 10(33), 77-87.

Hernández, O. F. (2014). La investigación formativa y la profesionalización docente. En Hernández, O. F.; V. S. Ramírez (eds.). La investigación formativa, retos y experiencias en la profesionalización docente. México: Porrúa Print.

Lozano, A. I.; M. F. Murillo Dasso (2014). La enseñanza de la historia de la arquitectura en el nivel primario para reconocer y valorar el patrimonio cultural propio. En VI Encuentro de Docentes e Investigadores en Historia del Diseño, la Arquitectura y la Ciudad "Iván Hernández Larguía". La Plata, Argentina.

Martín Bayona, N. (2015). La mota y los niños, un proyecto educativo para trabajar y acercar el patrimonio local a la educación infantil. Tesis de grado. Granada: Universidad de Granada.

Monereo, C. (2013). Research on University Teacher Education: Towards an Integrative Perspective. Journal for the Study of Education and Development, 36(3), 281-291. httos://doi.org/10.1174/021037013807533052 
Noyola, G. E. (2018). Formación de profesores de la educación especial: gestores de ambientes de aprendizaje. México: Ediciones del Lirio.

Pagés, J.; A. Santisteban (2010). La enseñanza y el aprendizaje del tiempo histórico en la educación primaria. Cuadernos CEDES, 30(82), 281-309.

https://doi.org/10.1590/S0101-32622010000300002

Reyes, W.; R. González (2007). Aproximación de la fundamentación teórica de un modelo de formación docente constructivista. Omnia, 13(3), 131-154.

Reyna, M.; N. C. Obregón; G. F. Ahumada (2018). La reflexión de la práctica docente en la formación inicial de profesores. En Pérez Herrera, M. E. (ed.). Una mirada introspectiva a la formación docente. México: Ediciones del lirio / BECENE.

Rodríguez Guerrero, J. P. (2019). La apropiación del Patrimonio Histórico Cultural por los niños de tercer grado de educación primaria a través de estrategias didácticas. Tesis México: BECENE. https://repositorio.becenes/p.edu.mx/jspui/handle/20.500.12584/307

Ruiz Hernández, C. (2015). Hacia una comprobación experimental de la zona de desarrollo próximo de Vygotsky. CIENCIA Ergo-Sum: Revista científica multidisciplinaria de la Universidad Autónoma del Estado de México, 22(2), 167-171.

Saxe, G. B. (2015). Studying Culture-Cognition Relations in Collective Practices of Daily Life: A Research Framework / El estudio de las relaciones cultura-cognición en las prácticas colectivas cotidianas: un modelo de investigación. Infancia y Aprendizaje, 38(3), 473-508.

SEP (2012). San Luis Potosí. La entidad donde vivo. México: SEP. https://issuu.com/sbasica/docs/san-luis-potosi

Solís, N. P. (2019). Los niños y el patrimonio. Los guardianes en el pueblo mágico de Real de Catorce, San Luis Potosí, México. Revista de El Colegio de San Luis, 9(19), 271-293.

Veraksa, N.; O. Shiyan; I. Shiyan; N. Pramling; I. Pramling-Samuelsson (2016). Comunicación entre maestro y niño en educación infantil: teoría y práctica educativa vygotskiana. Revista para el Estudio de Educación y Desarrollo, 39(2), 221-243.

https://www.tandfonline.com/doi/pdf/10.1080/02103702.2015.1133091?needAccess=true

Vosniadou, S. (2013). Model Based Reasoning and the Learning of Counter-Intuitive Science Concepts. Infancia y Aprendizaje, 36(1), 5-33.

Vygotsky, L. (1979). Interacciones entre aprendizaje y desarrollo. El desarrollo de los procesos psicológicos superiores. España: Crítica-Grijalbo, 123-140. 